\title{
Insight into the human Cyp24a1 inhibition by VID-400: docking and molecular dynamics studies
}

\author{
Abhimati Shukla \\ BIITP Trainee, Biotech Consortium India Limited, New Delhi,India \\ E mail: abhimati.shukla1991@gmail.com
}

\begin{abstract}
The 1, 25-dihydroxyvitamin D3 24-hydroxylase, is an enzyme that in humans is encoded by the CYP24A1gene. The P450s are considered to have a central role in chemical carcinogenesis and are involved in tumor initiation and promotion as they can activate or deactivate many carcinogens. Furthermore, the P450s can influence the response of established tumors to anti-cancer drugs as $\mathrm{P} 450$ s are involved in the metabolism of several anti-cancer drugs. CYP24A1 protein, member of Cytochrome P450 family which contains more than 50 enzymes, is found expressed in kidney and also in other tissues both healthy and cancerous. The CYP24A1 protein exists in two states-open state and closed state both of which will be studied in this research. Both open and closed state of CYP24A1 were first modeled and further docked with VID-400, an imidazole derivative seen to be a specific inhibitor of CYP24A1, and also with the inhibitors obtained after ligand based searching using VID-400 as the query in the PubChem database. The docking interactions for the inhibitors which gave positive results were further validated using Molecular dynamics simulation. The transition from open to closed form was also analyzed by comparing results of MD simulation of the ligand-free form and ligand-bound form of CYP24A1 and its transition to a closed binding pocket after the binding of the inhibitor.
\end{abstract}

Keywords:CYP450;CYP24A1;vitamin D;tetralones.

\section{Introduction}

The cytochrome P450 enzymes are the heme - thiolate proteins generally containing heme in the binding pocket. [1] The CYP24A1 enzyme, member of CYP450 family, has vitamin D3 substrate. In the case of humans vitamin D existsas either Vitamin D2 or Vitamin D3. These forms are further converted to 25-hydroxyvitamin $\mathrm{D}$ in liver and then to 25- di- hydroxy vitamin $\mathrm{D}$ (calcitriol) in the kidney.

It has been seen that vitamin D reduces the risk of cancer, and CYP24A1 is involved in the conversion of active vitamin $\mathrm{D}$ to inactive form thus increasing the risk of formation of cancerous cell in an individual. Due to this behavior of CYP24A1 enzyme, it has been considered an ideal target for diseases linked to vitamin D deficiency, including kidney diseases and cancer particularly prostate, colon, cervical, ovarian, esophageal, lung and basalcell carcinomas. [2]

The metabolism and conversion of active calcitriol, to inactive form can be slowed down by application of CYP24 inhibitors. The different categories of CYP24 inhibitors are azole, vitamin-D and tetralones. The azole compounds bind directly to heme iron through a lone pair of electrons from the heterocyclic nitrogen. These include VID-400,ketoconazole, liarazole, etc. The compounds of vitamin D like inhibitors include calcitriol, sulfone, sulfoximine, imidazole, etc. and those of tetralones include genestein. [3] 
This article focuses on the prediction of the inhibition of CYP24A1 enzymes using VID-400, an azole inhibitor, and the conformational changes occurring in the structure of CYP24A1 from ligand-free to ligand-bound form.

\section{Materials and Methods}

\section{Homology modeling and structural characterization:}

The aminoacid sequence for CYP24A1 (Homo sapiens) was obtained from UniProt database (Q07973_CP24A_HUMAN) and was aligned with structures deposited in the Protein Data Bank (PDB) using BLASTp algorithm. Crystal structures of ligand-free CYP24A1 (Rattus norvegicus) with PDB id 3K9V and ligand-bound CYP11A1 (Homo sapiens) with PDB id $3 \mathrm{NAO}$ were chosen as templates. The 50 homology models were generated using single template alignment approach with MODELLER 9v1.4 using the target-template alignments file obtained by ClustalW tool of EMBL-EBI and further modeled using the python script followed by structure optimization. The optimized models were determined for phi and psi angles using SAVES server.

The binding site of the CYP24A1 (Homo sapiens) was predicted using CastP server and also on the basis of the previous available data on CYP24A1 enzyme. The predictions made were further superimposed with the template structure i.e. CYP24A1 (Rattus norvegicus) and thus the binding pocket was validated.

\section{Docking studies with VID-400:}

The partial charges were assigned on VID-400, azole inhibitor for CYP24A1, and the protein molecule CYP24A1 using AutoDock tools of AutoDock4.0. The HEME group of the receptor file i.e. CYP24A1 was processed separately and the PDBQT files were generated with a $+\mathbf{3}$ charge on Fe. A grid with $\mathbf{7 4} * \mathbf{7 0} * \mathbf{8 0}$ grid points and spacing center $\mathbf{0 . 3 7 5 \AA}$ centered on the active site residues including heme group was further generated using AutoGrid 4.0 tools. The AutoDock 4.0 program was used for docking ligand VID-400 on the active site using Lamarckian Genetic algorithm, consisting of 100 runs. Conformations with high binding energy were further validated using MD simulation.

3D based ligand search in PubChem database was performed using the structure of VID-400, a selective inhibitor for CYP24A1 enzyme, as the reference structure and the compounds with $95 \%$ similarity were searched. The 18 compounds were further filtered on the basis of the Tanimato values. The obtained 7 compounds were further docked in the same conditions as used for the compound VID-400.

\section{MD simulation studies:}

All simulations were carried out with the help of Gromacs4.5. CYP24A1 was processed under Gromos43a1 force field. The charges on Heme group were taken from the study reported by Ferla et al. in 2014. Force field parameters for VID-400 were generated by the ProDRG server. Complexes were solvated in a cubic box containing SPC water model. Prior to minimization solvated systems were neutralized by adding $\mathrm{Na}^{+}$and $\mathrm{Cl}^{-}$ions at $0.1 \mathrm{M}$ concentration. Minimization of the molecule was carried out for 3000 steps to release conflicting contacts followed with restrained dynamics simulation which was done in two phases- one at constant volume and temperature (EqNVT) and other at constant pressure and temperature (EqNPT). Both NVT and NPT equilibration were carried out for 5ns using Velocity-rescale algorithm for tcoupl in NVT and Velocity-rescale algorithm for tcoupl and Parrinello-Rahman algorithm for pcoupl in NPT. Production MD run was then performed for 10ns using Parinello- Rahman and Velocity-rescale algorithm for pcoupl and tcoupl respectively. 
Energy fluctuations and RMSD of the complex was analyzed with respect to simulation time. The backbone root mean square fluctuations (RMSF) for each residue were monitored. The inter-molecular interactions i.e. interaction between heme and azole ring was assessed for stability of docking complex.

\section{Analysis of conformational changes in CYP24A1:}

In a research by Tereza Hendrychova et al in 2012 on open to close transition of CYP450 enzymes was studied. It was seen that the binding pockets of different CYP variants like, CYP2B4, CYP2D6, CYP2A6, CYP2C9, attained a closed form after the binding of the inhibitor. Similarly, the open to close transition of CYP24A1 (Homo sapiens) was seen after MD simulation for 10ns. The conformational changes occurring in the open form of CYP24A1 after the binding of the inhibitors were further analyzed by calculating the radius of gyration. According to the frames, snapshots of the protein were generated for every $2 \mathrm{~ns}$ for carrying out further analysis.

\section{Results:}

\section{Homology modeling and structural characterization:}

Homology models of CYP24A1 apo and holo conformation, i.e. the homology models for the ligand free form and ligand bound form, were constructed based on the crystal structures of CYP24A1 (Rattus norvegicus) and CYP11A1 (Homo sapiens), showing Ident value as $\mathbf{8 5 \%}$ and 39\% respectively, in the blastP analysis of the UniProt sequence of the CYP24A1 (Homo sapiens) sequence.

The pair-wise alignments for the target and template sequences generated by the Clustal W tool are shown in the

Figure 1(a) and 1(b) given below:

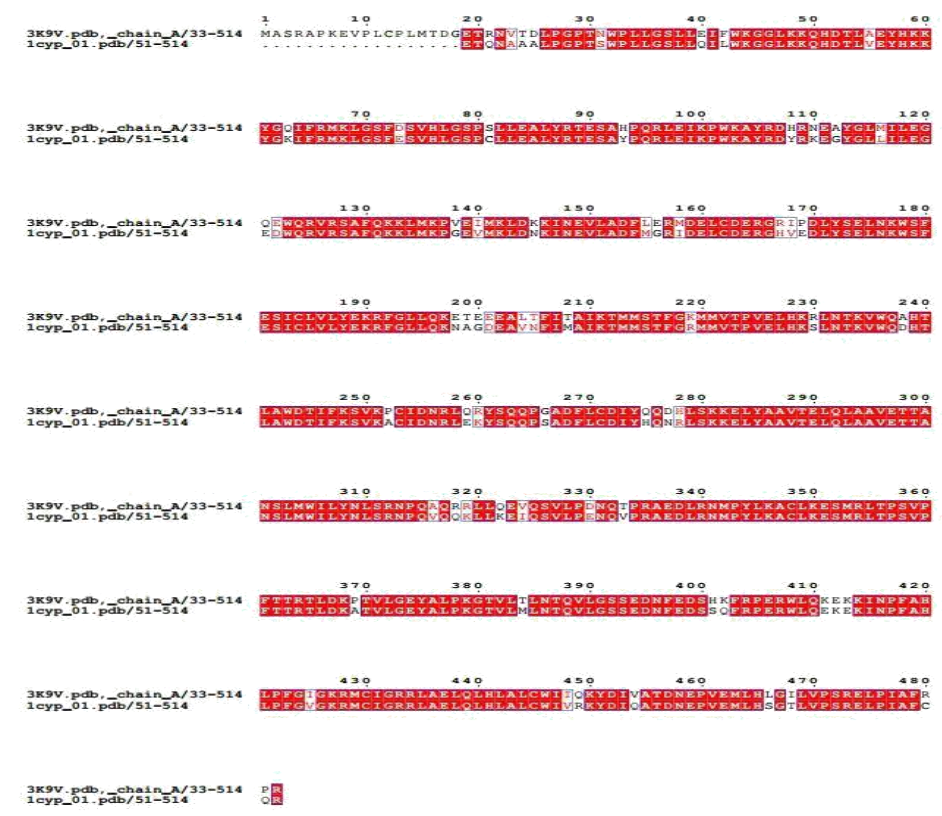

Figure 1(a): alignment of the CYP24A1 (Homo sapiens) shown at the bottom of the alignment with the template used for modeling of open form CYP24A1 (Rattus norvegicus) shown at the top of the alignment. The identical regions are highlighted in red and the similar regions are marked in blue. The residues of both the sequences are numbered with the gap of 
The homology models were generated using the target-template alignments obtained for both the ligand-free and ligand-bound forms in which the 50 missing residues were omitted in both the cases for the 3D model building. The homology models thus generated using modeler are shown in the Figure 2 shown below.

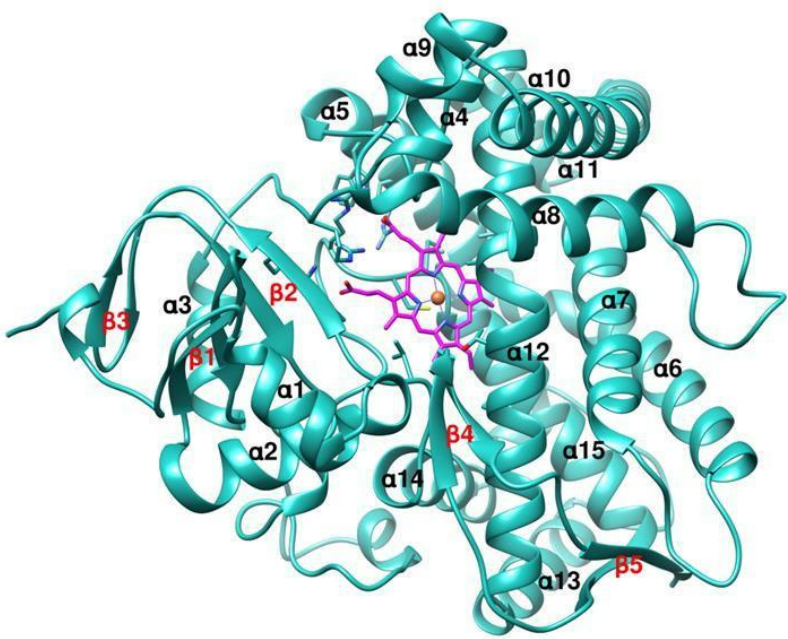

Figure 2(a)

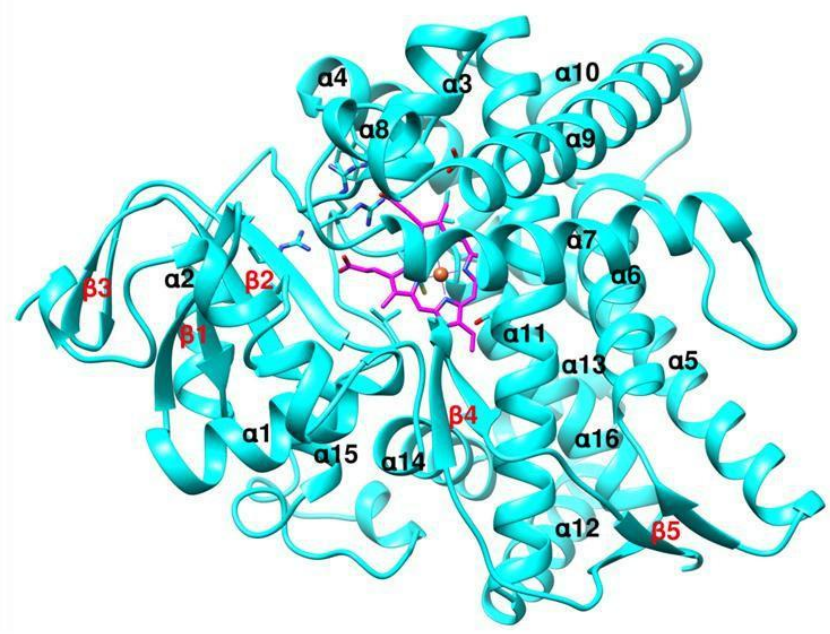

Figure 2(b)

Figure 2 (a) the model for open conformation generated using CYP24A1 (Rattus norvegicus)

Figure 2 (b) the model for close conformation generated using CYP11A1 (Homo sapiens)

The models generated were further validated using Ramachandran plot analysis and CYP24A1 ligand-free and ligand-bound forms revealed $0.2 \%$ of the residues individually in the disallowed regions.
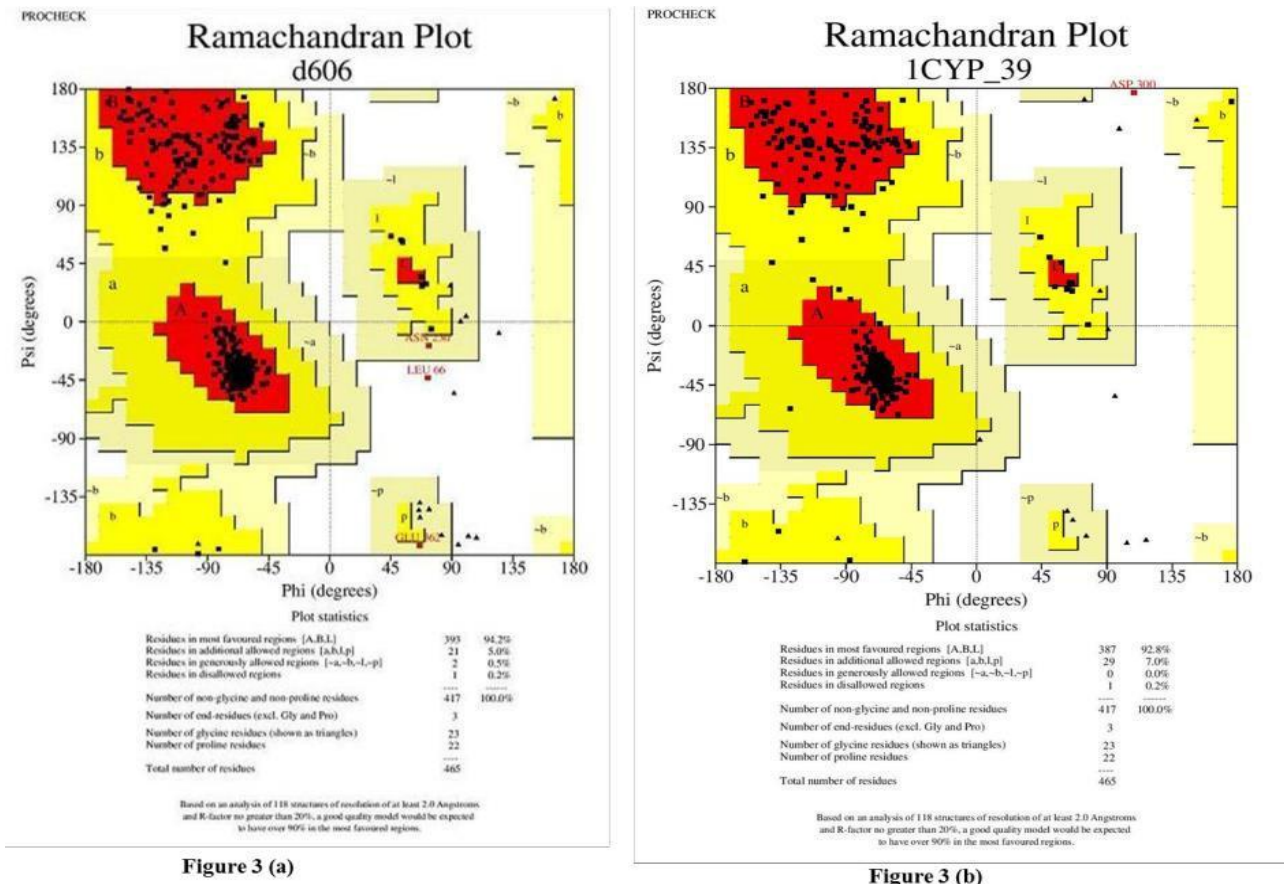

The target and template structures were compared using the visualizer Chimera 1.6.2 which gave 0.154 $\AA$ RMSD for CYP24A1 (Rattus norvegicus) and CYP24A1 (Homo sapiens) for ligand-free conformation and $\mathbf{0 . 1 9 6 \AA ~ R M S D ~ f o r ~ C Y P 1 1 A 1 ~ ( H o m o ~ s a p i e n s ) ~ a n d ~ C Y P 2 4 A 1 ~}$ (Homo sapiens) for ligand-bound conformation. Further, the ligand-free and ligand-bound conformations were superimposed to study the movement of helices and sheets responsible for the two forms and it was found that the helices $\boldsymbol{\alpha} 7$ and $\boldsymbol{\alpha 9}$ of ligand-free conformation 
showed large deviations from the helices $\alpha 8^{*}$ and $\boldsymbol{\alpha 1 0 *}$ of ligand-bound conformation and are thus largely responsible for formation of the two forms of CYP24A1.

\section{Docking studies with VID-400:}

The azole compound VID-400 was docked on the models selected for the ligand-free and ligand-bound forms of CYP24A1 after Ramachandran plot analysis. The relative binding energies obtained were $\mathbf{- 5 . 7 9}$ and $\mathbf{- 8 . 4 5} \mathbf{k c a l} / \mathbf{m o l}$ for the ligand-free and ligand-bound forms respectively and the interaction of $\mathrm{N}$ - heterocyclic ring of azole with iron in the Heme domain was seen to be $\mathbf{3 . 0 7 7} \AA$ in ligand-free conformation and $1.893 \AA$ in ligand-bound conformation. It was clearly seen that the ligand interacts comparatively more efficiently in the case of ligand-bound conformation than in ligand-free conformation.

The conformations generated were seen to interact with the HEME ring of CYP24A1 and with the surrounding residues - Arg 128, Ile 131, Trp 134, Leu 148, Met 246, Phe 249, Ala 326, Thr 330, Phe 393, Thr 394, Thr 395, Gly 499, Thr 500. The docked conformation of CYP24A1 (open and close form) is shown in Figure 4 shown below.

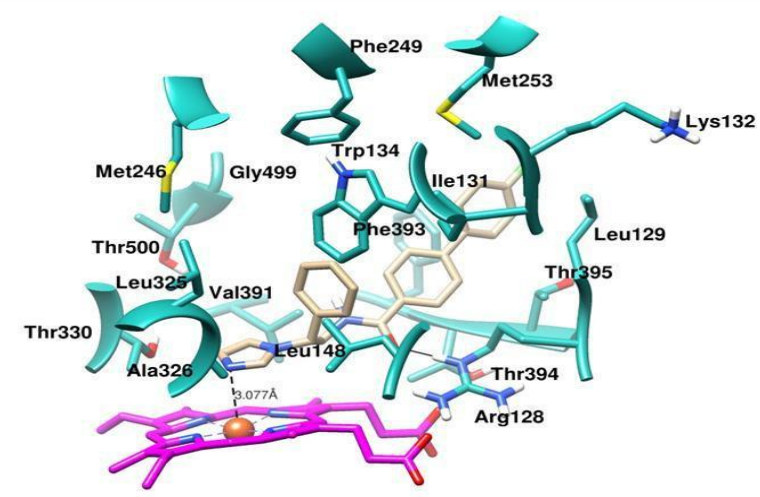

Figure 4 (a)

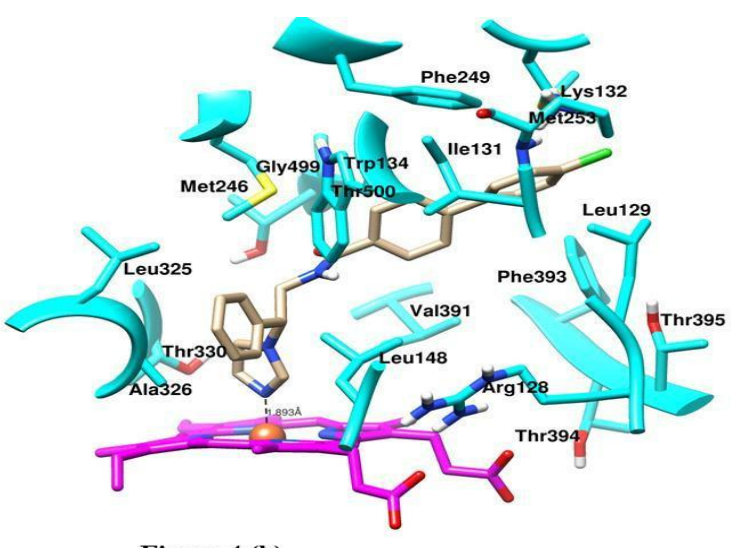

Figure 4 (b)

\section{Simulation:}

The stability of VID-400 and CYP24A1 complex was further validated by 10ns molecular dynamics simulation of the docked structural conformations obtained. The distance between $\mathrm{Fe}$ of HEME ring and the azole ring reduced to $\mathbf{1 . 7 0 0 ~} \AA$ in the case of ligand-free conformation and $\mathbf{1 . 8 0 6} \AA$ in the case of ligand-bound conformation. The binding mode adapted by VID-400 in the binding site in both the conformation is shown in Figure $\mathbf{5}$ shown below.
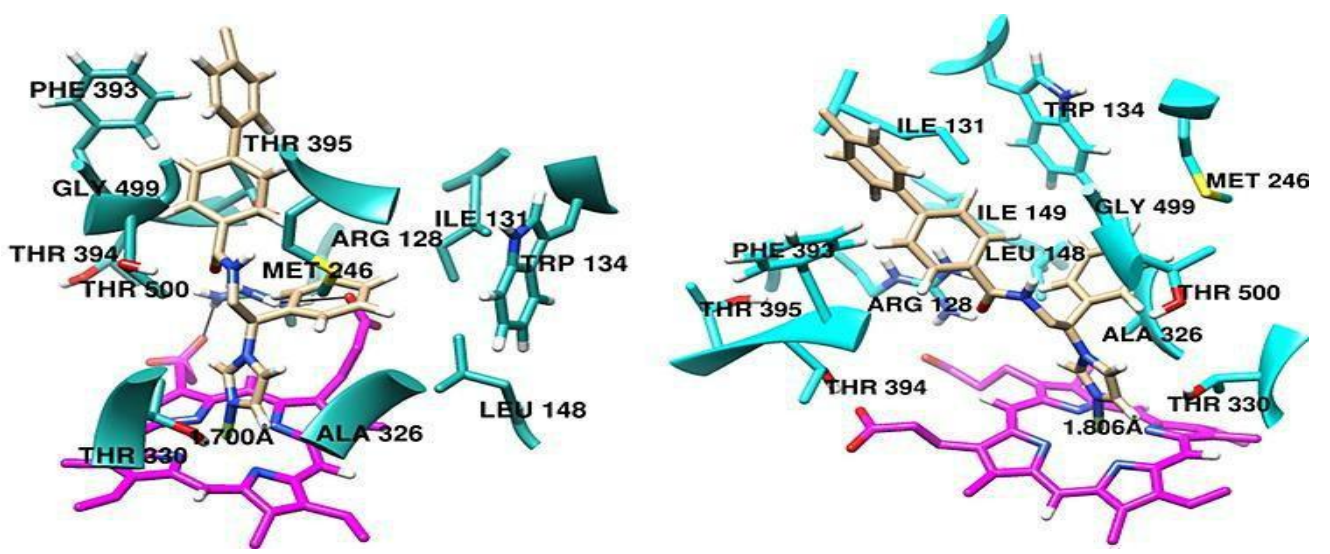

Figure 5: VID-400 simulated complex in ligand-free and ligand-bound form: HEME ring is shown in magenta and binding site residues in cyan for open conformation and light sea green for close conformation. 
The comparison of the conformations attained by VID-400 in both ligand-free and ligandbound conformation is show in Figure 6 below. In the figure it can be seen that the conformation attained in forms of CYP24A1 were identical to one another.

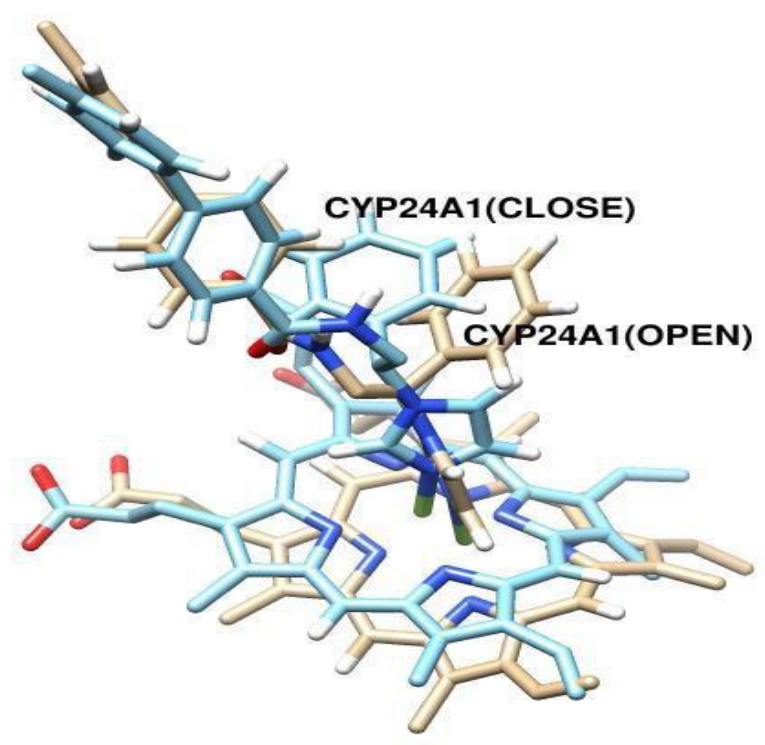

Figure 6: Superimposed image of conformations of VID-400 in open and closed form attained after MD Simulation:conformation for closed form is shown in blue and open form in creme.

The MD simulation results provided exact binding interaction of the docked complex. The RMSD for CYP24A1 backbone was originated and plotted which revealed that the complex was relatively stable throughout the simulation. The overall RMSD range for backbone atoms were 0.0004-0.355nm for open and $\mathbf{0 . 0 0 0 4 - 0 . 3 1 n m}$ for close conformation. A comparative view for the RMSD values in both ligand-free and ligand-bound conformation is shown in Figure 7 shown below.

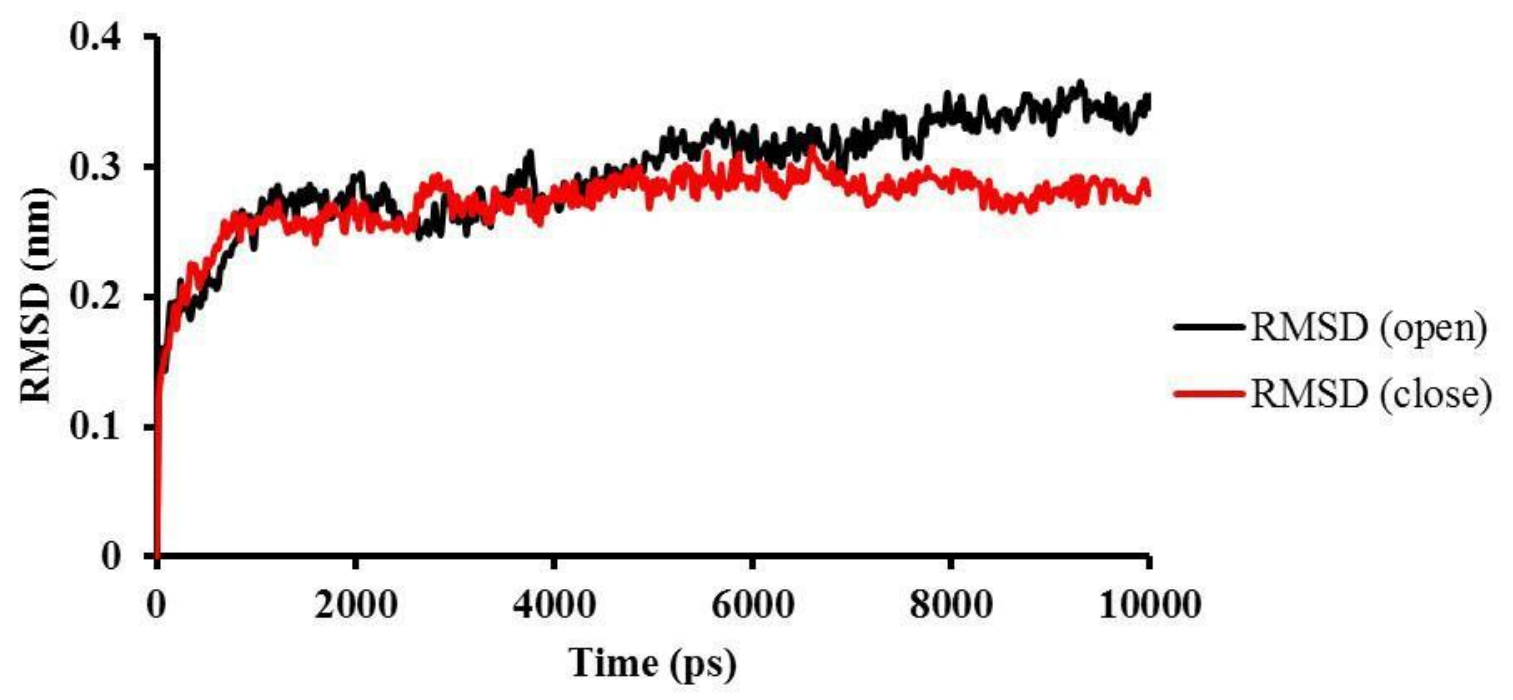

Figure 7: RMSD (backbone) open and close conformation for CYP24A1 
The RMSF values of backbone were assessed for each residue of CYP24A1. Maximum backbone fluctuations up to $\mathbf{0 . 4 2 9} \mathbf{n m}$ for ligand-free form was observed at GLU (234 residue) and for ligand-bound form was observed at PRO (297 residue) of $0.2987 \mathrm{~nm}$. The binding site residues showed the RMSF values ranging between $0.053 \mathbf{n m - 0 . 1 9 4 n m}$ of which maximum fluctuation was seen in residues THR $255(0.194 \mathrm{~nm})$, LEU $79(0.175 \mathrm{~nm})$ and PHE 455(0.150 nm). The RMSF plot for the backbone of ligand-free and ligand-bound conformation is shown in Figure 8 below.

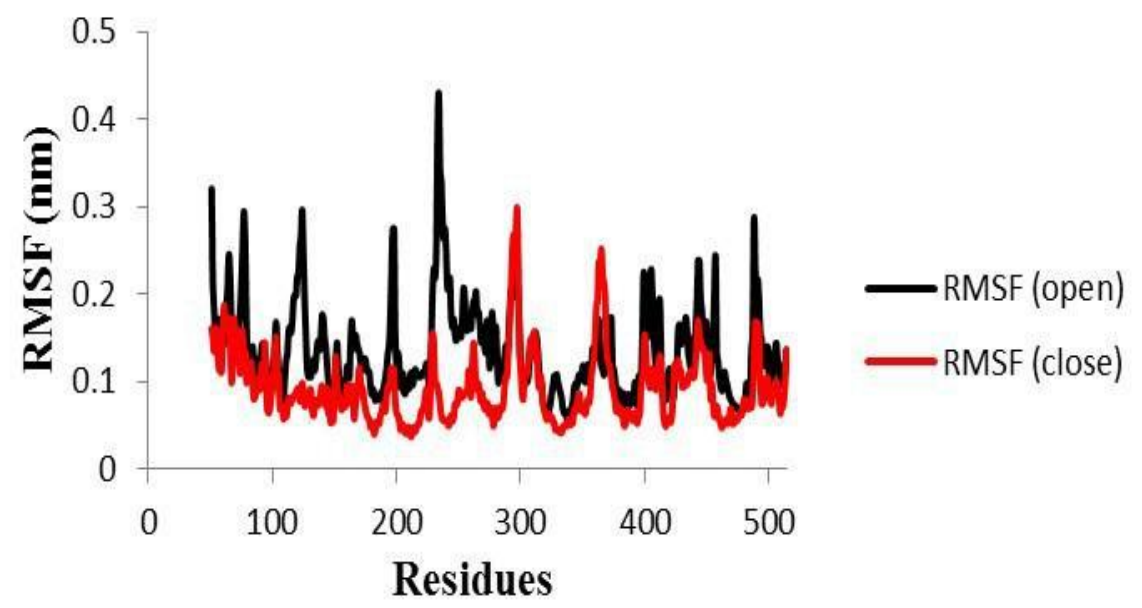

Figure 8: RMSF (backbone) open and close conformation

The plot for no. of H-bonds between protein and the ligand was assessed for both ligand-free and ligand-bound conformation and is shown in Figure 9 given below.
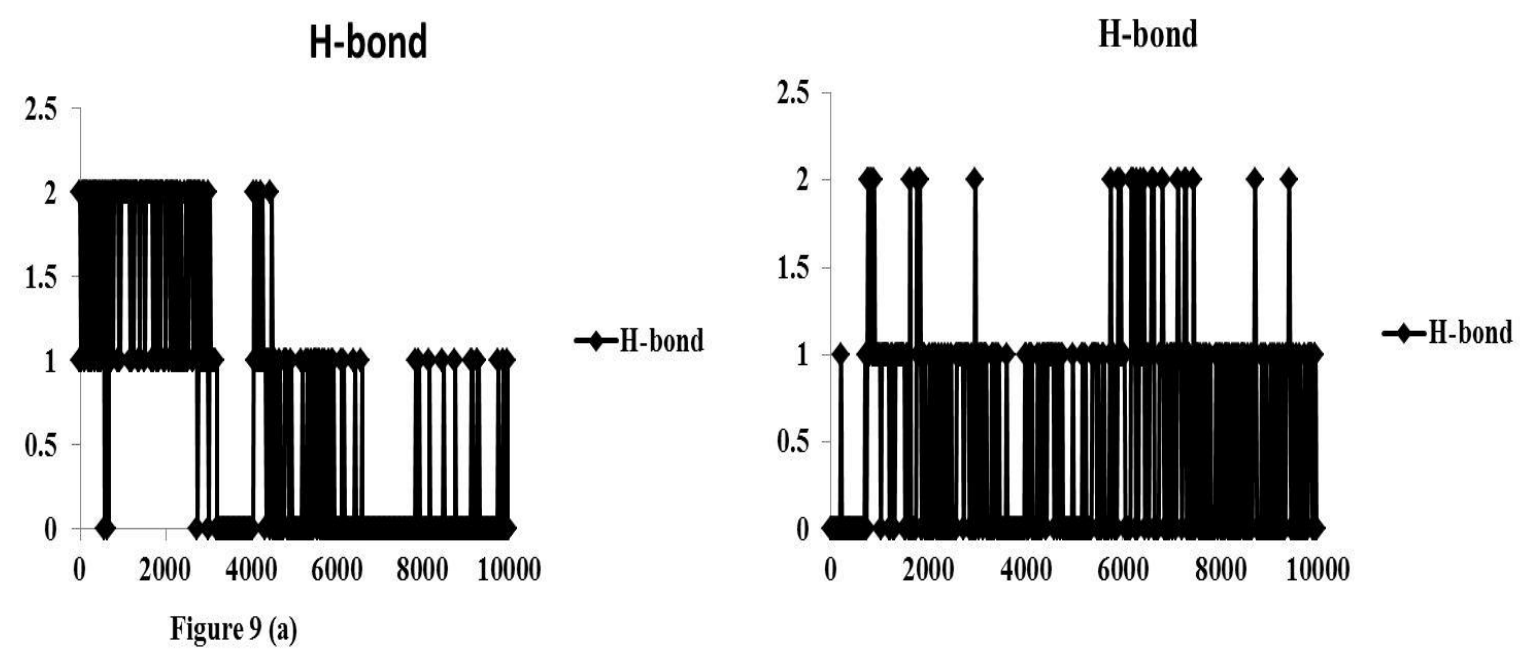

Figure 9: 9(a) no. of H-bonds between protein and ligand for open conformation 9(b) no.of $\mathrm{H}$ - bonds between protein and ligand for close conformation.

The Fe-N distance was plotted for both ligand-free and ligand-bound conformation which revealed that the distance was stable in the case of ligand-bound conformation whereas in the case of ligand-free conformation, large fluctuations were seen from 4000ps to 6000ps which stabilized thereafter. 

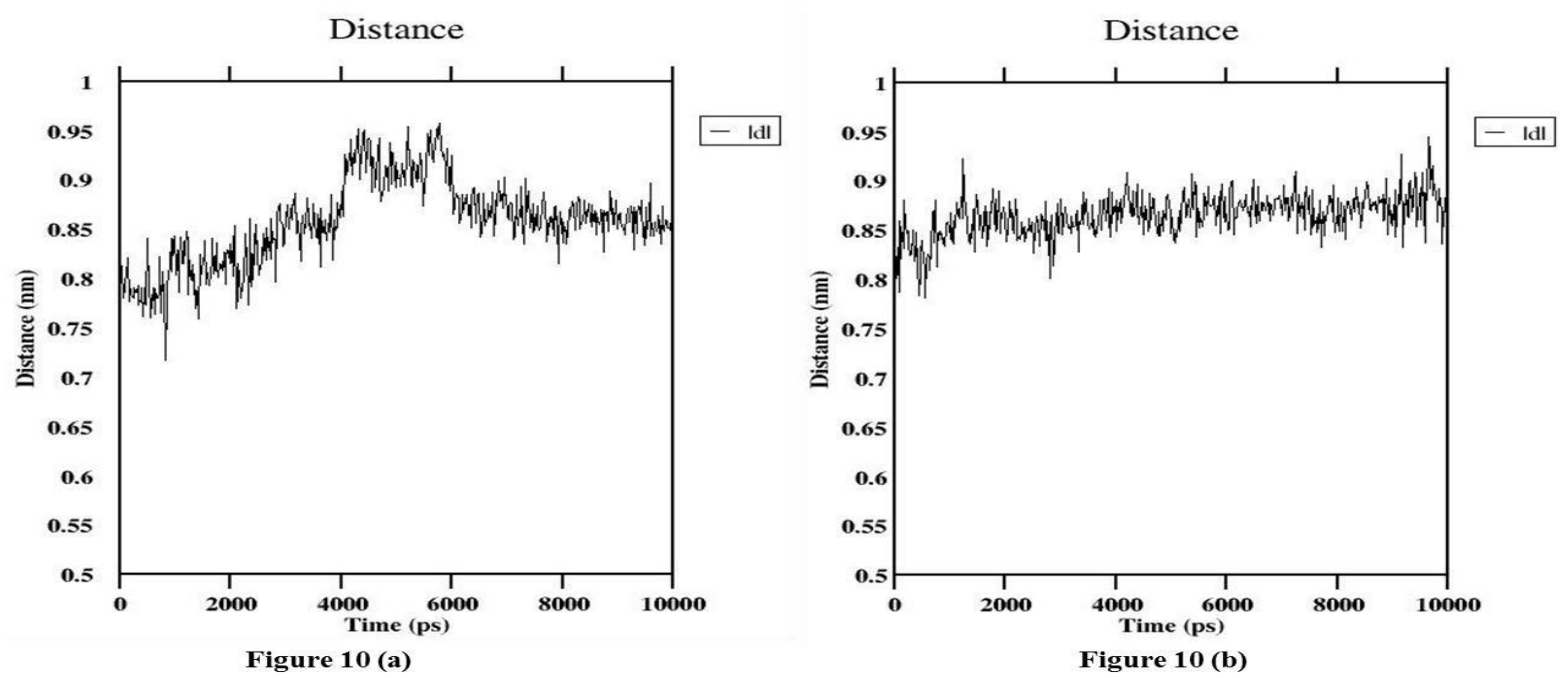

Figure 10: 10(a) shows the distance between the HEME ring of ligand-free conformation and VID-400 10(b) shows the distance between the HEME ring of ligand-bound conformation and VID-400

The binding energy after simulation was generated using g_pbsa system in which it was seen that after simulation the binding energy of VID-400 increased in comparison to the energy obtained after docking. A comparison of the docked and simulated energies is shown in the Table 1 given below

Table 1: Binding energy result

\begin{tabular}{|c|c|c|c|}
\hline \multicolumn{2}{|c|}{ Binding energy for CYP } & \multicolumn{2}{c|}{ Binding energy for CYP } \\
24A1(open) $(\mathrm{kcal} / \mathrm{mol})$ & 24A1(closed) $(\mathrm{kcal} / \mathrm{mol})$ \\
\hline Docked & Simulated & Docked & Simulated \\
\hline-5.98 & -57.52 & -8.45 & -56.24 \\
\hline
\end{tabular}

\section{Conformational analysis}

Compactness of the ligand-free conformation was monitored by the comparative gyration plot for both ligand-free and ligand-bound conformation which revealed that the ligand-free conformation underwent folding and attained a stable structure which is somewhat like the ligand-bound conformation after the binding of the inhibitor.

Radius of gyration

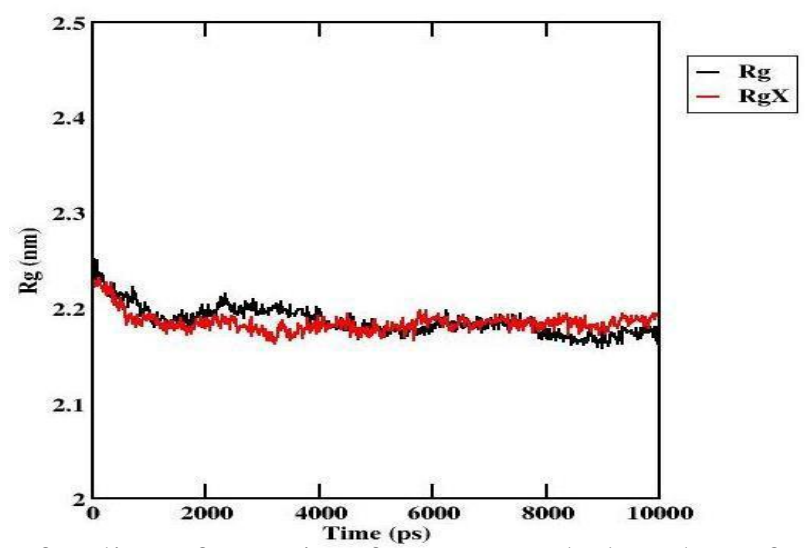

Figure 11: Comparison of radius of gyration for open and closed conformation: the black lines show the radius of gyration for open form and the red lines show the radius of gyration for close form 
The conformational changes were further monitored every 2000ps which show that the binding site which was open at 0ps gradually attained a closed conformation at 10000ps. This phenomenon basically occurred due to the large deviations in the positions of the helices $\alpha 7$ and $\alpha 9$ and towards their position in the ligand bound form shown by $\alpha 8^{*}$ and $\alpha 10^{*}$ as shown in the Figure 12(a) and 12(b).
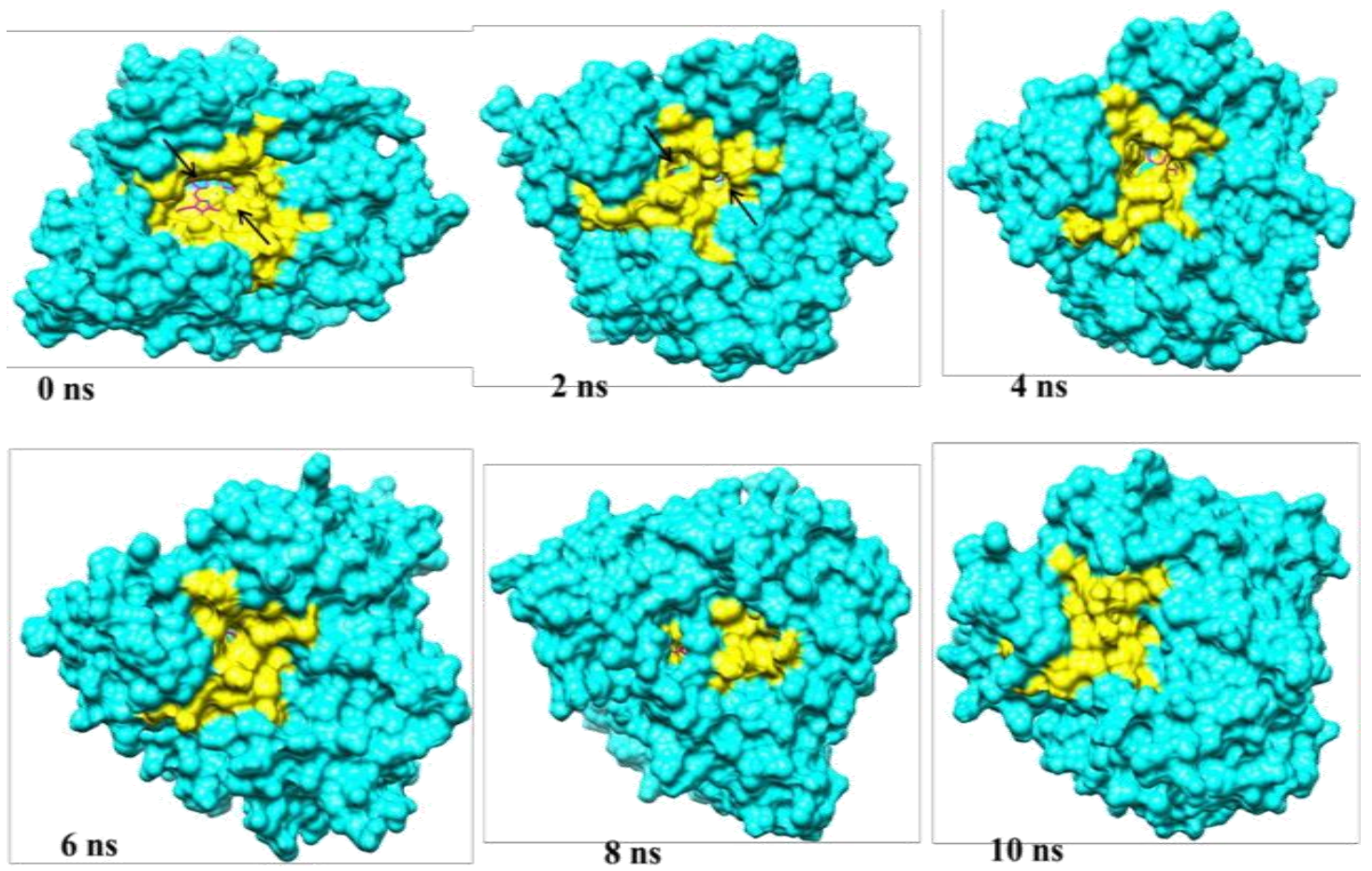

Figure 12: Conformational changes in active site of open conformation after binding of ligand: the protein is shown in surface view with cyan color and the active site residues in yellow with arrows showing the movement of helices

\section{Discussion:}

The homology model generated for CYP24A1 (Homo sapiens) in previous researches were at lesser identity like Gomaa et al in 2007 generated a model building and docking studies using three human structures-CYP2C8, CYP2C9 and CYP3A4 as templates with percent sequence identity ranging between 25 to $26 \%$ where as the templates used in this research were at higher sequence identity of $85 \%$ and $35 \%$ for the ligand-free and ligand-bound form respectively. In the study by Gomaa et al in 2007 , the models generated were for the ligandbound form of CYP24A1 and no analysis of the ligand-free form was made. In the study by Ferla et al in 2014, the model structure was generated using CYP24A1 (Rattus norvegicus) as template which was also used in our research for generating the open conformation of CYP24A1 (Homo sapiens).

The MD simulation of CYP24A1 (Homo sapiens) with VID-400 has not been reported till date. In a research by Ferla et al in 2014, MD simulation of CYP24A1 (Homo sapiens) with imidazole derivatives was conducted for $5 \mathrm{~ns}$ but no further analysis for the transitions of the holo i.e. ligand free form and apo i.e. ligand-bound form was done.

\section{Conclusion:}

The CYP24A1 enzymes, member of the Cytochrome P450 family, are the mitochondrial enzymes involved in drug metabolism and have also been recognized as cancer targets. The crystal structure of the target has yet not been coined so the 3D structure was modeled using 
different templates for both open and closed forms. The binding site was then characterized for docking of the inhibitor VID-400.

The docking revealed the interaction of the HEME group with the azole ring of the inhibitor with good binding energies. Keeping VID-400 as a reference, the similar inhibitors were then searched in the PubChem database yielding a list of compounds whose binding capacity was then monitored in the active site. The confirmations with good binding energies and interacting in a similar manner as VID-400 were then extracted.

The simulation of the confirmations obtained was then carried out for the validation of the results obtained. In the end we concluded that VID-400 shows good binding affinity for the CYP24A1 enzyme and that the two forms of the CYP24A1 enzyme are inter-convertible as after simulation of the complex it was seen that the binding cavity of the protein which was open in the case of the open conformation, closed gradually and attained a conformation which was somewhat identical to the close conformation.

It was also seen that the confirmation of the VID-400 in the open form was different from that attained in the case of close conformation, but after simulation the confirmations came out to be identical in both forms.

\section{References:}

1.Reichhart, D.K., et. al., 2000. "Cytochrome P450: A success story" Genome Biol., https://doi.org/10.1186/gb-2000-1-6-reviews3003

2.Arnaud, St. R., 2011. "CYP24A1: Structure, function and physiological role Vitamin D" Vitamin D, https://doi.org/10.1016/B978-0-12-381978-9.10004-6

3.Watson and Ronald R., 2013. "Handbook of Vitamin D in Human Health: Prevention, Treatment and Toxicity".

4.Annalora A. J., et al., 2010. "Crystal structure of CYP24A1, a mitochondrial cytochrome P450 involved in vitamin D metabolism”, J Mol Biol doi: 10.1016/j.jmb.2009.11.057.

5.Strushkevich, N., et al., 2011. "Structural basis for pregnenolone biosynthesis by the mitochondrial monooxygenase system”, Proc Natl Acad Sci USA doi: 10.1073/pnas.1019441108

6.Sali, A. and Blundell, T. L., 1993. "Comparative protein modelling by satisfaction of spatial restraints”, J Mol Biol DOI: 10.1006/jmbi.1993.1626

7.http://www.ebi.ac.uk/Tools/msa/clustalw2 /

8.Laskowski, R. A. et al., 1993. "PROCHECK - a program to check the stereochemical quality of protein structures", J. App. Cryst, https://doi.org/10.1107/S0021889892009944

9.Dundas, J., et. al., 2006. "CASTp: computed atlas of surface topography of proteins with structural and topographical mapping of functionally annotated residues" Nucl.Acids Res, DOI: $10.1093 / \mathrm{nar} / \mathrm{gk} 1282$

10.Masuda, S., et. al., 2006. "Generation of a homology model for the human cytochrome P450, CYP24A1, and the testing of putative substrate binding residues by site-directed mutagenesis and enzyme activity studies" Archives of Biochemistry and Biophysics, DOI:10.1016/j.abb.2006.11.030 
11.Gomaa, M. S., et al., 2006. "Homology model of 1 ,25-dihydroxyvitamin D324- hydroxylase cytochrome P450 24A1 (CYP24A1): Active site architecture and ligand binding "Journal of Steroid Biochemistry \& Molecular Biology DOI: 10.1016/j.jsbmb.2006.09.041.

12.Ferla, S., et. al., 2014. "Small-Molecule Inhibitors of 25-Hydroxyvitamin D- 24- Hydroxylase (CYP24A1): Synthesis and Biological Evaluation" Journal of Medicinal Chemistry DOI:10.1021/jm5009314

13.Morris, G. M., et al., 1998. "Automated docking using a Lamarckian genetic algorithm and an empirical binding free energy function" J Comput Chem DOI: 10.1002/(SICI)1096987X(19981115)19:14<1639::AID-JCC10>3.0.CO;2-B.

14.https://pubchem.ncbi.nlm.nih.gov/

15.Helms, V. and Wade, R.C., 1995 "Thermodynamics of water mediating protein-ligand interactions in Cytochrome 450cam: A molecular dynamics study", Biophy. J. 1995 doi: 10.1016/S0006-3495(95)79955-6

16.Lounnas, V. and Wade, R.C., 1997. "The exceptionally stable salt-bridges in cytochrome P450cam have functional roles", Biochem. J. 1997 DOI:10.1021/bi9622940

17.Lindahl, E., et al., Gromacs User Manual version 4.5.4.

18.Schüttelkopf, A. W. and Van Aalten, D. M., (2004). "PRODRG: a tool for high-throughput crystallography of protein-ligand complexes", Acta Crystallogr D Biol Crystallogr DOI:10.1107/S0907444904011679

19.Tereza, H., et al., 2012. "Dynamics and Hydration of the Active sites of Mammalian Cytochrome P450 probed by Molecular Dynamics Simulations" CentralDrug Metabolism DOI:10.2174/138920012798918408 